\title{
TIME-OPTIMAL BOUNDARY CONTROL OF AN INFINITE ORDER PARABOLIC SYSTEM WITH TIME LAGS
}

\author{
ADAM KOWALEWSKI*, ANNA KRAKOWIAK ** \\ * Institute of Automatics, AGH University of Science and Technology \\ Al. Mickiewicza 30, 30-059 Cracow, Poland \\ e-mail:ako@ia.agh.edu.pl \\ ** Institute of Mathematics, Technical University of Cracow \\ ul. Warszawska 24, 31-155 Cracow, Poland \\ e-mail: skrakowi@riad.usk.pk.edu.pl
}

\begin{abstract}
In this paper the time-optimal boundary control problem is presented for a distributed infinite order parabolic system in which time lags appear in the integral form both in the state equation and in the boundary condition. Some specific properties of the optimal control are discussed.
\end{abstract}

Keywords: time-optimal boundary control, infinite order, parabolic system, time lags.

\section{Introduction}

Distributed parameter systems with delays can be used to describe many phenomena in the real world. As is well known, heat conduction, properties of elastic-plastic material, fluid dynamics, diffusion-reaction processes, the transmission of the signals at a certain distance by using electric long lines, etc., all lie within this area. The object that we are studying (temperature, displacement, concentration, velocity, etc.) is usually referred to as the state.

We are interested in the case where the state satisfies proper differential equations that are derived from basic physical laws, such as Newton's law, Fourier's law, etc. The space in which the state exists is called the state space, and the equation that the state satisfies is called the state equation. In particular, we are interested in the cases where state equations are one of the following types: partial differential equations, integro-differential equations, or abstract evolution equations.

Equations with deviating arguments appeared in Euler's works. However, systematic research of such equations began only in the 20th century, as a result of the development of applied sciences and particularly automatic control theory. Consequently, equations with deviating arguments are a well-known mathematical tool for representing many physical problems. Historically, they have achieved great popularity among mathematicians, physists, and engineers.

During the last twenty years, equations with deviating arguments have been applied not only in applied mathematics, physics and automatic control, but also in some problems of economy and biology. Currently, the theory of equations with deviating arguments constitutes a very important subfield of mathematical control theory.

Consequently, equations with deviating arguments are widely applied in optimal control problems of distributed parameter systems with time delays.

Various optimization problems associated with the optimal control of distributed parabolic systems with time delays appearing in the boundary conditions were studied recently in (El-Saify, 2005; El-Saify, 2006; Knowles, 1978; Kowalewski, 1988; Kowalewski, 1990a; Kowalewski, 1990b; Kowalewski, 1993; Kowalewski, 1998; Kowalewski, 1999; Kowalewski, 2001; Kowalewski and Duda, 1992; Kowalewski and Krakowiak, 1994; Kowalewski and Krakowiak, 2000; Kowalewski and Krakowiak, 2006; Wang, 1975).

In (Wang, 1975), optimal control problems for second order parabolic systems with Neumann boundary conditions involving constant time delays were considered. Such systems constitute in a linear approximation, a universal mathematical model for many diffusion pro- 
cesses in which time-delayed feedback signals are introduced at the boundary of a system spatial domain. For example, in the area of plasma control, it is of interest to confine a plasma in a given bounded spatial domain $\Omega$ by introducing a finite electric potential barrier or a "magnetic mirror" surrounding $\Omega$. For a collision-dominated plasma (Kowalewski and Duda, 1992), its particle density is describable by a second order parabolic equation. Due to particle inertia and the finiteness of the electric potential barrier or the magnetic mirror field strength, the particle reflection at the domain boundary is not instantaneous. Consequently, the particle flux at the boundary of $\Omega$ at any time depends on the flux of particles which escaped earlier and reflected back into $\Omega$ at a later time. This leads to Neumann boundary conditions involving time delays. Necessary and sufficient conditions which optimal controls must satisfy were derived. Estimates and a sufficient condition for the boundedness of solutions were obtained for second order parabolic systems with specified forms of feedback controls.

Subsequently, in (Knowles, 1978), time-optimal control problems of linear second order parabolic systems with Neumann boundary conditions involving constant time delays were considered. Using the results of (Wang, 1975), the existence of a unique solution of such parabolic systems was discussed. A characterization of the optimal control in terms of the adjoint system is given. This characterization was used to derive specific properties of the optimal control (bang-bangness, uniqueness, etc.). These results were also extended to some cases of the nonlinear control without convexity and to certain fixed time problems.

Consequently, in (Kowalewski, 1988; Kowalewski, 1990a; Kowalewski, 1990b; Kowalewski, 1993; Kowalewski, 1998; Kowalewski, 1999; Kowalewski, 2001) and (Kowalewski and Duda, 1992), linear quadratic problems for second order parabolic systems with time delays given in a different form (constant time delays, time-varying delays, time delays given in the integral form, etc.) were presented. Moreover, in (Kowalewski and Krakowiak, 1994) and (Kowalewski and Krakowiak, 2000), time-optimal distributed control problems of second order parabolic systems with constant and integral time lags appearing both in state equations and Neumann boundary conditions were investigated.

Finally, in (El-Saify, 2005; El-Saify, 2006), linear quadratic optimal distributed and boundary control problems for $n \times n$ second order and $n \times n$ infinite order parabolic time-varying lag systems were considered.

In particular, in (Kowalewski and Krakowiak, 2006), time-optimal boundary control problems for second order parabolic systems with deviating arguments appearing in an integral form both in state equations and in Neumann boundary conditions were considered. The presented minimum time problem can be generalized to the case of in- finite order time delay parabolic systems.

For this reason, in this paper we consider the timeoptimal boundary control problem for a linear infinite order parabolic system in which time lags appear in an integral form both in the state equation and in the Neumann boundary condition. Such an infinite order parabolic system can be treated as a generalization of the mathematical model for a plasma control process.

The existence and uniqueness of solutions of such a parabolic equation are proved. The optimal control is characterized by the adjoint equation. Using this characterization, particular properties of the time-optimal boundary control are proved, i.e., bang-bangness, uniqueness, etc.

\section{Preliminaries}

Let $\Omega$ be a bounded open set of $\mathbb{R}^{n}$ with a smooth boundary $\Gamma$. We define the infinite order Sobolev space $H^{\infty}\left\{a_{\alpha}, 2\right\}(\Omega)$ of functions $\Phi(x)$ defined on $\Omega$ (Dubinskii, 1975; Dubinskii, 1976) as follows:

$$
\begin{aligned}
& H^{\infty}\left\{a_{\alpha}, 2\right\}(\Omega) \\
& =\left\{\Phi(x) \in C^{\infty}(\Omega): \sum_{|\alpha|=0}^{\infty} a_{\alpha}\left\|\mathcal{D}^{\alpha} \Phi\right\|_{2}^{2}<\infty\right\},
\end{aligned}
$$

where $C^{\infty}(\Omega)$ is the space of infinite differentiable functions, $a_{\alpha} \geq 0$ is a numerical sequence, $\|\cdot\|_{2}$ is the norm in the space $L^{2}(\Omega)$, and

$$
\mathcal{D}^{\alpha}=\frac{\partial^{|\alpha|}}{\left(\partial x_{1}\right)^{\alpha_{1}} \ldots\left(\partial x_{n}\right)^{\alpha_{n}}},
$$

$\alpha=\left(\alpha_{1}, \ldots, \alpha_{n}\right)$ being a multi-index for differentiation, $|\alpha|=\sum_{i=1}^{n} \alpha_{i}$.

The space $H^{-\infty}\left\{a_{\alpha}, 2\right\}(\Omega)$ (Dubinskii, 1975; Dubinskii, 1976) is defined as the formal conjugate space to the space $H^{\infty}\left\{a_{\alpha}, 2\right\}(\Omega)$, namely,

$$
\begin{aligned}
& H^{-\infty}\left\{a_{\alpha}, 2\right\}(\Omega) \\
& =\left\{\Psi(x): \Psi(x)=\sum_{|\alpha|=0}^{\infty}(-1)^{|\alpha|} a_{\alpha} \mathcal{D}^{\alpha} \Psi_{\alpha}(x)\right\},
\end{aligned}
$$

where $\Psi_{\alpha} \in L^{2}(\Omega)$ and $\sum_{|\alpha|=0}^{\infty} a_{\alpha}\left\|\Psi_{\alpha}\right\|_{2}^{2}<\infty$.

The duality pairing of the spaces $H^{\infty}\left\{a_{\alpha}, 2\right\}(\Omega)$ and $H^{-\infty}\left\{a_{\alpha}, 2\right\}(\Omega)$ is postulated by the formula

$$
\langle\Phi, \Psi\rangle=\sum_{|\alpha|=0}^{\infty} a_{\alpha} \int_{\Omega} \Psi_{\alpha}(x) \mathcal{D}^{\alpha} \Phi(x) \mathrm{d} x,
$$

where $\Phi \in H^{\infty}\left\{a_{\alpha}, 2\right\}(\Omega), \Psi \in H^{-\infty}\left\{a_{\alpha}, 2\right\}(\Omega)$.

From above, $H^{\infty}\left\{a_{\alpha}, 2\right\}(\Omega)$ is everywhere dense in $L^{2}(\Omega)$ with topological inclusions and $H^{-\infty}\left\{a_{\alpha}, 2\right\}(\Omega)$ denotes the topological dual space with respect to $L^{2}(\Omega)$, so we have the following chain of inclusions:

$$
H^{\infty}\left\{a_{\alpha}, 2\right\}(\Omega) \subseteq L^{2}(\Omega) \subseteq H^{-\infty}\left\{a_{\alpha}, 2\right\}(\Omega) .
$$




\section{Existence and uniqueness of solutions}

Consider now the distributed-parameter system described by the following parabolic delay equation:

$$
\begin{aligned}
& \frac{\partial y}{\partial t}+A y+\int_{a}^{b} c(x, t) y(x, t-h) \mathrm{d} h=u, \\
& x \in \Omega, \quad t \in(0, T), \\
& y\left(x, t^{\prime}\right)=\Phi_{0}\left(x, t^{\prime}\right), \quad x \in \Omega, \quad t^{\prime} \in[-b, 0), \\
& y(x, 0)=y_{0}(x), \quad x \in \Omega, \\
& \frac{\partial y}{\partial \eta_{A}}=\int_{a}^{b} d(x, t) y(x, t-h) \mathrm{d} h+v, \\
& x \in \Gamma, \quad t \in(0, T), \\
& y\left(x, t^{\prime}\right)=\Psi_{0}\left(x, t^{\prime}\right), \quad x \in \Gamma, \quad t^{\prime} \in[-b, 0),
\end{aligned}
$$

where $\Omega$ has the same properties as in Section 2. We have

$$
\begin{array}{rlrlrl}
y & \equiv y(x, t ; u), & u & \equiv u(x, t), & v & \equiv v(x, t), \\
Q & \equiv \Omega \times(0, T), & \bar{Q} & =\bar{\Omega} \times[0, T], & Q_{0}=\Omega \times[-b, 0), \\
\Sigma & =\Gamma \times(0, T), & \Sigma_{0} & =\Gamma \times[-b, 0), & &
\end{array}
$$

$T$ is a specified positive number representing a time horizon, $c$ is a given real $C^{\infty}$ function defined on $\bar{Q}, d$ is a given real $C^{\infty}$ function defined on $\Sigma, h$ is a time lag such that $h \in(a, b)$ and $a>0, \Phi_{0}$ and $\Psi_{0}$ are initial functions defined on $Q_{0}$ and $\Sigma_{0}$, respectively.

The operator $\frac{\partial}{\partial t}+A$ in the state equation (6) is an infinite order parabolic operator, $A$ is given by

$$
A y=\sum_{|\alpha|=0}^{\infty}(-1)^{|\alpha|} a_{\alpha} \mathcal{D}^{2 \alpha} y(x, t)
$$

and

$$
\sum_{|\alpha|=0}^{\infty}(-1)^{|\alpha|} a_{\alpha} \mathcal{D}^{2 \alpha}
$$

is an infinite order elliptic partial differential operator (Dubinskii, 1981).

Equations (6)-(10) constitute a Neumann problem. The left-hand side of (9) is written in the following form:

$$
\begin{array}{r}
\frac{\partial y}{\partial \eta_{A}}=\sum_{|w|=0}^{\infty}\left(\mathcal{D}^{w} y(v)\right) \cos \left(n, x_{i}\right)=q(x, t), \\
x \in \Gamma, \quad t \in(0, T),
\end{array}
$$

where $\partial y / \partial \eta_{A}$ is the normal derivative at $\Gamma$, directed towards the exterior of $\Omega, \cos \left(n, x_{i}\right)$ is the $i$-th direction cosine of $n$, with $n$ being the normal at $\Gamma$ to the exterior to $\Omega$, and

$$
\begin{aligned}
q(x, t)= & \int_{a}^{b} \mathrm{~d}(x, t) y(x, t-h) \mathrm{d} h \\
& +v(x, t), \quad x \in \Gamma, \quad t \in(0, T) .
\end{aligned}
$$

First we shall prove sufficient conditions for the existence of a unique solution of the mixed initial-boundary value problem (6)-(10) for the case where the boundary control $v \in L^{2}(\Sigma)$.

For this purpose, we introduce the Sobolev space $H^{\infty, 1}(Q)$ (Lions and Magenes, 1972, Vol. 2, p. 6) defined by

$$
\begin{aligned}
H^{\infty, 1}(Q)= & H^{0}\left(0, T ; H^{\infty}\left\{a_{\alpha}, 2\right\}(\Omega)\right) \\
& \cap H^{1}\left(0, T ; H^{0}(\Omega)\right),
\end{aligned}
$$

which is a Hilbert space normed by

$$
\left(\int_{0}^{T}\|y(t)\|_{H^{\infty}\left\{a_{\alpha}, 2\right\}(\Omega)}^{2} \mathrm{~d} t+\|y\|_{H^{1}\left(0, T ; H^{0}(\Omega)\right)}^{2}\right)^{1 / 2}
$$

where the space $H^{1}\left(0, T ; H^{0}(\Omega)\right)$ is defined in Chapter 1 of (Lions and Magenes, 1972, Vol. 1).

The existence of a unique solution for the mixed initial-boundary value problem (6)-(10) on the cylinder $Q$ can be proved using a constructive method, i.e., first, solving (6)-(10) on the subcylinder $Q_{1}$ and in turn on $Q_{2}$, and so on, until the procedure covers the whole cylinder $Q$. In this way, the solution in the previous step determines the next one.

For simplicity, we introduce the following notation:

$$
\begin{aligned}
E_{j} & \triangleq((j-1) a, j a), & Q_{j} & =\Omega \times E_{j}, \\
Q_{0} & =\Omega \times[-b, 0), & \Sigma_{j} & =\Gamma \times E_{j}, \\
\Sigma_{0} & =\Gamma \times[-b, 0) & j & =1, \ldots, K .
\end{aligned}
$$

Using Theorem 15.2 of (Lions and Magenes, 1972, Vol. 2, p. 81) we can prove the following lemma:

Lemma 1. Let

$$
\begin{gathered}
u \in\left(H^{\infty, 1}(Q)\right)^{\prime}, \quad v \in L^{2}(\Sigma), \\
f_{j} \in\left(H^{\infty, 1}\left(Q_{j}\right)\right)^{\prime},
\end{gathered}
$$

where

$$
\begin{aligned}
f_{j}(x, t)=u(x, t)-\int_{a}^{b} c(x, t) y_{j-1}(x, t-h) \mathrm{d} h & \\
y_{j-1}(\cdot,(j-1) a) & \in H^{\infty}\left\{a_{\alpha}, 2\right\}(\Omega) \\
q_{j} & \in L^{2}\left(\Sigma_{j}\right)
\end{aligned}
$$


where

$$
q_{j}(x, t)=\int_{a}^{b} d(x, t) y_{j-1}(x, t-h) \mathrm{d} h+v(x, t) .
$$

Then there exists a unique solution $y_{j} \in H^{\infty, 1}\left(Q_{j}\right)$ for the mixed initial-boundary value problem (6), (9), (19).

Proof. We observe that for $j=1$,

$$
\left.y_{j-1}\right|_{Q_{0}}(x, t-h)=\Phi_{0}(x, t-h)
$$

and

$$
\left.y_{j-1}\right|_{\Sigma_{0}}(x, t-h)=\Psi_{0}(x, t-h) .
$$

Then the assumptions (18), (19) and (20) are fulfilled if we assume that $\Phi_{0} \in H^{\infty, 1}\left(Q_{0}\right), y_{0} \in H^{\infty}\left\{a_{\alpha}, 2\right\}(\Omega)$, and $\Psi_{0} \in L^{2}\left(\Sigma_{0}\right)$. These assumptions are sufficient to ensure the existence of a unique solution $y_{1} \in H^{\infty, 1}\left(Q_{1}\right)$. In order to extend the result to $Q_{2}$, we have to prove that $y_{1}(\cdot, a) \in H^{\infty}\left\{a_{\alpha}, 2\right\}(\Omega),\left.\quad y_{1}\right|_{\Sigma_{1}} \in L^{2}\left(\Sigma_{1}\right)$ and $f_{2} \in\left(H^{\infty, 1}\left(Q_{2}\right)\right)^{\prime}$. Really, from Theorems 2.1 and 2.2 of (Kowalewski, 1998), $y_{1} \in H^{\infty, 1}\left(Q_{1}\right)$ implies that the mapping $t \rightarrow y_{1}(\cdot, t)$ is continuous from $[0, a] \rightarrow$ $H^{\infty}\left\{a_{\alpha}, 2\right\}(\Omega)$. Thus, $y_{1}(\cdot, a) \in H^{\infty}\left\{a_{\alpha}, 2\right\}(\Omega)$. Then using the trace theorem (Theorem 2.3 of (Kowalewski, 1998)) we can verify that $y_{1} \in H^{\infty, 1}\left(Q_{1}\right)$ implies that $\left.y_{1} \rightarrow y_{1}\right|_{\Sigma_{1}}$ is a linear, continuous mapping of $H^{\infty, 1}\left(Q_{1}\right) \rightarrow H^{\infty, 1}\left(\Sigma_{1}\right)$. Thus, $\left.y_{1}\right|_{\Sigma_{1}} \in L^{2}\left(\Sigma_{1}\right)$. Also it is easy to notice that the assumption (18) follows from the fact that $y_{1} \in H^{\infty, 1}\left(Q_{1}\right)$ and $u \in\left(H^{\infty, 1}(Q)\right)^{\prime}$. Then, there exists a unique solution $y_{2} \in H^{\infty, 1}\left(Q_{2}\right)$. The foregoing result is now summarized for $j=3, \ldots, K$.

Theorem 1. Let $y_{0}, \Phi_{0}, \Psi_{0}, v$ and $u$ be given with $y_{0} \in H^{\infty}\left\{a_{\alpha}, 2\right\}(\Omega), \Phi_{0} \in H^{\infty, 1}\left(Q_{0}\right), \Psi_{0} \in L^{2}\left(\Sigma_{0}\right)$, $v \in L^{2}(\Sigma)$ and $u \in\left(H^{\infty, 1}(Q)\right)^{\prime}$. Then there exists a unique solution $y \in H^{\infty, 1}(Q)$ for the mixed initialboundary value problem (6)-(10). Moreover, $y(\cdot, j a) \in$ $H^{\infty}\left\{a_{\alpha}, 2\right\}(\Omega)$ for $j=1, \ldots, K$.

\section{Problem formulation. Optimization theorems}

Now, we shall formulate the minimum-time problem for (6)-(10) in the context of Theorem 1 i.e.,

$$
v \in U=\left\{v \in L^{2}(\Sigma):|v(x, t)| \leq 1\right\} .
$$

We shall define the reachable set $Y$ such that

$$
Y=\left\{y \in L^{2}(\Omega):\left\|y-z_{d}\right\|_{L^{2}(\Omega)} \leq \epsilon\right\},
$$

where $z_{d}, \epsilon$ are given with $z_{d} \in L^{2}(\Omega)$ and $\epsilon>0$.

Solving the stated minimum-time problem is equivalent to hitting the target set $Y$ in minimum time, that is, minimizing the time $t$, for which $y(t ; v) \in Y$ and $v \in U$.
Moreover, we make the following assumption:

There exists $T>0$ and $v \in U$ with $y(T ; v) \in Y$.

Theorem 2. If the assumption (23) holds, then the set $Y$ is reached in minimum time $t^{*}$ by an admissible control $v^{*} \in U$. Moreover,

$$
\int_{\Omega}\left(z_{d}-y\left(t^{*} ; v^{*}\right)\right)\left(y\left(t^{*} ; v\right)-y\left(t^{*} ; v^{*}\right)\right) \mathrm{d} x \leq 0,
$$

Outline of the proof: Let us define the following set:

$$
t^{*}=\inf \{t: y(t ; v) \in Y \text { for some } v \in U\} \text {. }
$$

The minimum is well defined, as (23) guarantees that this set is nonempty. By definition, we can choose $t_{n} \downarrow t^{*}$ and admissible controls $\left\{v_{n}\right\}$ such that

$$
y\left(t_{n} ; v_{n}\right) \in Y, \quad n=1,2,3, \ldots
$$

Each $v_{n}$ is defined on $\Gamma \times\left(0, t_{n}\right) \supset \Gamma \times\left(0, t^{*}\right)$. To simplify the notation, we denote the restriction of $v_{n}$ to $\Gamma \times\left(0, t^{*}\right)$ again by $v_{n}$. The set of admissible controls then forms a weakly compact, convex set in $L^{2}\left(\Gamma \times\left(0, f^{*}\right)\right.$, and so we can extract a weakly convergent subset $\left\{v_{m}\right\}$, which converges weakly to some admissible control $v^{*}$.

Consequently, Theorem 1 implies that $y(t ; v) \in$ $H^{\infty}\left\{a_{\alpha}, 2\right\}(\Omega) \subset L^{2}(\Omega)$ for each $v \in L^{2}(\Sigma)$ and $t>0$. Then using Theorem 1.2 of (Lions, 1971, p. 102) and Theorem 1 it is easy to verify that the mapping $v \rightarrow y\left(t^{*} ; v\right)$, from $L^{2}\left(\Gamma \times\left(0, t^{*}\right)\right)$ into $L^{2}(\Omega)$, is continuous. Since any continuous linear mapping between Banach spaces is also weakly continuous (Dunford and Schwartz, 1958), Theorem V. 3.15, the affine mapping $v \rightarrow y\left(t^{*} ; v\right)$ must also be weakly continuous. Hence,

$$
y\left(t^{*} ; v_{m}\right) \rightarrow y\left(t^{*} ; v^{*}\right) \text { weakly in } L^{2}(\Omega) .
$$

Moreover,

$$
\frac{\mathrm{d} y(v)}{\mathrm{d} t} \in L^{2}\left(\left[0, t^{*}\right], H^{0}(\Omega)\right),
$$

for each $v \in U$, by definition of $H^{\infty, 1}\left(\Omega \times\left(0, t^{*}\right)\right)$, and

$$
\begin{aligned}
& \left\|y\left(t_{m} ; v_{m}\right)-y\left(t^{*} ; v_{m}\right)\right\|_{L^{2}(\Omega)} \\
& =\left\|\int_{t^{*}}^{t_{m}} \dot{y}\left(\sigma ; v_{m}\right) \mathrm{d} \sigma\right\|_{L^{2}(\Omega)} \\
& \leq \sqrt{t_{m}-t^{*}}\left(\int_{t^{*}}^{t_{m}}\left\|\dot{y}\left(\sigma ; v_{m}\right)\right\|_{L^{2}(\Omega)}^{2} \mathrm{~d} \sigma\right)^{1 / 2} .
\end{aligned}
$$


Applying Theorem 1.2 of (Lions, 1971) and Theorem 1 again, the set $\left\{\dot{y}\left(v_{m}\right)\right\}$ must be bounded in $L^{2}\left(0, t^{*} ; H^{0}(\Omega)\right)$, and so

$$
\left\|y\left(t_{m} ; v_{m}\right)-y\left(t^{*} ; v_{m}\right)\right\|_{L^{2}(\Omega)} \leq M \sqrt{t_{m}-t^{*}} .
$$

Combining (27) and (29) shows that

$$
\begin{aligned}
& y\left(t_{m} ; v_{m}\right)-y\left(t^{*} ; v^{*}\right) \\
& =\left(y\left(t_{m} ; v_{m}\right)-y\left(t^{*} ; v_{m}\right)\right) \\
& \quad+\left(y\left(t^{*} ; v_{m}\right)-y\left(t^{*} ; v^{*}\right)\right)
\end{aligned}
$$

converges weakly to zero in $L^{2}(\Omega)$, and therefore $y\left(t^{*} ; v^{*}\right) \in Y$ as $Y$ is closed and convex, hence weakly closed. This shows that $Y$ is reached in time $t^{*}$ by an admissible control accordingly, $t^{*}$ must be the minimum time and $v^{*}$ an optimal control.

We shall now prove the second part of our theorem. Indeed, from Theorem 3.1 (Lions and Magenes, 1972, Vol. 1, p. 19) $y(v) \in H^{\infty, 1}(Q)$ implies that the mapping $t \rightarrow y(t ; v)$, from $[0, T] \rightarrow H^{\infty}\left\{a_{\alpha}, 2\right\}(\Omega) \subset L^{2}(\Omega)$ is continuous for each fixed $v$, and so $y\left(t^{*} ; v\right) \notin$ int $Y$, for any $v \in U$, by the minimality of $t^{*}$.

From our earlier remarks, the set

$$
\mathcal{A}\left(t^{*}\right)=\left\{y\left(t^{*} ; v_{x}\right): v_{x} \in U\right\}
$$

is weakly compact and convex in $L^{2}(\Omega)$. Applying Theorem 21.11 of (Choquet, 1969) to the sets $\mathcal{A}\left(t^{*}\right)$ and $Y$ shows that there exists a nontrivial hyperplane $z^{\prime} \in L^{2}(\Omega)$ separating these sets, that is,

$$
\begin{aligned}
& \int_{\Omega} z^{\prime} y\left(t^{*} ; v\right) \mathrm{d} x \\
& \quad \leq \int_{\Omega} z^{\prime} y\left(t^{*} ; v^{*}\right) \mathrm{d} x \leq \int_{\Omega} z^{\prime} y \mathrm{~d} x
\end{aligned}
$$

for all $v \in U$ and $y \in L^{2}(\Omega)$ with

$$
\left\|y-z_{d}\right\|_{L^{2}(\Omega)} \leq \varepsilon .
$$

From the second inequality in (32), $z^{\prime}$ must support the set $Y$ at $y\left(t^{*} ; v^{*}\right)$. Since $L^{2}(\Omega)$ is a Hilbert space, $z^{\prime}$ must be of the form

$$
z^{\prime}=\lambda\left(z_{d}-y\left(t^{*} ; v^{*}\right)\right) \text { for some } \lambda>0
$$

Subsequently, dividing (32) by $\lambda$ gives the desired result (24).

\section{Optimization theorems}

We shall apply Theorem 2 to the control problem of (6)(10).
To simplify (24), we introduce the adjoint equation, and for every $v \in U$ we define the adjoint variable $p=$ $p(v)=p(x, t ; v)$ as the solution of the equation

$$
\begin{aligned}
& -\frac{\partial p(v)}{\partial t}+A^{*} p(v) \\
& \quad+\int_{a}^{b} c(x, t+h) p(x, t+h ; v) \mathrm{d} h=0, \\
& x \in \Omega, \quad t \in\left(0, t^{*}-b\right)
\end{aligned}
$$

$$
\begin{gathered}
-\frac{\partial p(v)}{\partial t}+A^{*} p(v) \\
+\int_{a}^{t^{*}-t} c(x, t+h) p(x, t+h ; v) \mathrm{d} h=0, \\
x \in \Omega, \quad t \in\left(t^{*}-b, t^{*}-a\right), \\
-\frac{\partial p(v)}{\partial t}+A^{*} p(v)=0, \quad x \in \Omega, t \in\left(t^{*}-a, t^{*}\right), \\
p\left(x, t^{*} ; v\right)=z_{d}(x)-y\left(x, t^{*} ; v\right), \quad x \in \Omega,
\end{gathered}
$$

$$
\begin{array}{r}
\frac{\partial p(v)}{\partial \eta_{A^{*}}}(x, t)=\int_{a}^{b} d(x, t+h) p(x, t+h ; v) \mathrm{d} h, \\
x \in \Gamma, \quad t \in\left(0, t^{*}-b\right),
\end{array}
$$

$$
\begin{gathered}
\frac{\partial p(v)}{\partial \eta_{A^{*}}}(x, t)=\int_{a}^{t^{*}-t} d(x, t+h) p(x, t+h ; v) \mathrm{d} h \\
x \in \Gamma, \quad t \in\left(t^{*}-b, t^{*}-a\right), \\
\frac{\partial p(v)}{\partial \eta_{A^{*}}}(x, t)=0, \quad x \in \Gamma, \quad t \in\left(t^{*}-a, t^{*}\right),
\end{gathered}
$$

where

$$
\left\{\begin{array}{l}
\frac{\partial p(v)}{\partial \eta_{A^{*}}}(x, t)=\sum_{|w|=0}^{\infty}\left(\mathcal{D}^{w} p(v)\right) \cos \left(n, x_{i}\right), \\
A^{*} p=\sum_{|\alpha|=0}^{\infty}(-1)^{|\alpha|} a_{\alpha} \mathcal{D}^{2 \alpha} p(x, t) .
\end{array}\right.
$$

Remark 1. If $t^{*}<b$, then we consider (36) and (40) on $\Omega \times\left(0, t^{*}-a\right)$ and $\Gamma \times\left(0, t^{*}-a\right)$, respectively.

The existence of a unique solution to the problem (35) - (41) on the cylinder $\Omega \times\left(0, t^{*}\right)$ can be proved using a constructive method. It is easy to notice that for given $z_{d}$ and $u$, the problem (35)-(41) can be solved backwards in time starting from $t=t^{*}$, i.e., first, solving (35)-(41) on the subcylinder $Q_{K}$ and in turn on $Q_{K-1}$, etc., until the procedure covers the whole cylinder $\Omega \times\left(0, t^{*}\right)$. For 
this purpose, we may apply Theorem 1 (with an obvious change of variables)

Hence, using Theorem 1, the following result can be proved:

Theorem 3. Let the hypothesis of Theorem $\square$ be satisfied. Then for given $z_{d} \in L^{2}(\Omega)$ and any $v \in L^{2}(\Sigma)$, there exists a unique solution $p(v) \in H^{\infty, 1}\left(\Omega \times\left(0, t^{*}\right)\right)$ for the adjoint problem (35)-(41).

We simplify the left-hand side of the inequality (24) using the adjoint equation (35)-(41). For this purpose, setting $v=v^{*}$ in (35)-(41), multiplying both sides of (35), (36), (37) by $y(v)-y\left(v^{*}\right)$, then integrating over $\Omega \times\left(0, t^{*}-b\right), \Omega \times\left(t^{*}-b, t^{*}-a\right)$ and $\Omega \times\left(t^{*}-a, t^{*}\right)$ respectively and then adding both sides of (35)-(37), we get

$$
\begin{aligned}
\int_{0}^{t^{*}} & \int_{\Omega}\left(-\frac{\partial p\left(v^{*}\right)}{\partial t}+A^{*} p\left(v^{*}\right)\right)\left(y(v)-y\left(v^{*}\right)\right) \mathrm{d} x \mathrm{~d} t \\
+ & \int_{0}^{t^{*}-b} \int_{\Omega}\left(\int_{a}^{b} c(x, t+h) p\left(x, t+h ; v^{*}\right) \mathrm{d} h\right) \\
& \times\left[y(x, t ; v)-y\left(x, t ; v^{*}\right)\right] \mathrm{d} x \mathrm{~d} t \\
+ & \left.\int_{t^{*}-b}^{t^{*}-a} \int_{\Omega}^{t^{*}-t} \int_{a} c(x, t+h) p\left(x, t+h ; v^{*}\right) \mathrm{d} h\right) \\
& \times\left[y(x, t ; v)-y\left(x, t ; v^{*}\right)\right] \mathrm{d} x \mathrm{~d} t \\
= & -\int_{\Omega} p\left(x, t^{*} ; v^{*}\right)\left(y\left(x, t^{*} ; v\right)-y\left(x, t^{*} ; v^{*}\right)\right) \mathrm{d} x \\
& +\int_{0}^{t^{*}} \int_{\Omega} p\left(v^{*}\right) \frac{\partial}{\partial t}\left(y(v)-y\left(v^{*}\right)\right) \mathrm{d} x \mathrm{~d} t \\
& +\int_{t^{*}-b} \int_{\Omega} \int_{a} c\left(y(x, t ; v)-y\left(x, t ; v^{*}\right)\right) \mathrm{d} h \mathrm{~d} x \mathrm{~d} t=0 . \\
+ & \int_{0} \int_{\Omega} A^{*} p\left(v^{*}\right)\left(y(v)-y\left(v^{*}\right)\right) \mathrm{d} x \mathrm{~d} t \\
+ & \int_{0}^{t^{*}-b} \int_{\Omega} \int_{a}^{b} c(x, t+h) p\left(x, t+h ; v^{*}\right) \\
& \left.\times(y, t ; v)-y\left(x, t ; v^{*}\right)\right) \mathrm{d} h \mathrm{~d} x \mathrm{~d} t \\
+ & t^{*}-t
\end{aligned}
$$

Then, applying (38), the formula (43) can be expressed as

$$
\begin{aligned}
& \int_{\Omega}\left(z_{d}-y\left(t^{*} ; v^{*}\right)\right)\left(y\left(t^{*} ; v\right)-y\left(t^{*} ; v^{*}\right)\right) \mathrm{d} x \\
& =\int_{0} \int_{\Omega} p\left(v^{*}\right) \frac{\partial}{\partial t}\left(y(v)-y\left(v^{*}\right)\right) \mathrm{d} x \mathrm{~d} t \\
& +\int_{0}^{t^{*}} \int_{\Omega} A^{*} p\left(v^{*}\right)\left(y(v)-y\left(v^{*}\right)\right) \mathrm{d} x \mathrm{~d} t \\
& +\int_{a}^{b} \int_{\Omega}^{t^{*}-b} \int_{0} c(x, t+h) p\left(x, t+h ; v^{*}\right) \\
& \quad \times\left(y(x, t ; v)-y\left(x, t ; v^{*}\right)\right) \mathrm{d} t \mathrm{~d} x \mathrm{~d} h \\
& +\int_{a}^{t^{*}-t} \int_{\Omega}^{t^{*}-a} \int_{t^{*}-b} c(x, t+h) p\left(x, t+h ; v^{*}\right) \\
& \quad \times\left(y(x, t ; v)-y\left(x, t ; v^{*}\right)\right) \mathrm{d} t \mathrm{~d} x \mathrm{~d} h .
\end{aligned}
$$

Using (6), the first integral on the right-hand side of (44) can be rewritten as

$$
\begin{aligned}
\int_{0}^{t^{*}} \int_{\Omega} p\left(v^{*}\right) \frac{\partial}{\partial t}\left(y(v)-y\left(v^{*}\right)\right) \mathrm{d} x \mathrm{~d} t \\
=-\int_{0}^{t^{*}} \int_{\Omega} p\left(v^{*}\right) A\left(y(v)-y\left(v^{*}\right)\right) \mathrm{d} x \mathrm{~d} t \\
-\int_{0}^{t^{*}} \int_{\Omega} p\left(x, t ; v^{*}\right)\left(\int_{a}^{b} c(x, t)\right. \\
\left.\quad \times\left(y(x, t-h ; v)-y\left(x, t-h ; v^{*}\right)\right) \mathrm{d} h\right) \mathrm{d} x \mathrm{~d} t \\
=-\int_{0}^{t^{*}} \int_{\Omega} p\left(v^{*}\right) A\left(y(v)-y\left(v^{*}\right)\right) \mathrm{d} x \mathrm{~d} t \\
-\int_{0}^{t^{*}} \int_{\Omega}^{b} p\left(x, t ; v^{*}\right) c(x, t) \\
\quad \times\left(y(x, t-h ; v)-y\left(x, t-h ; v^{*}\right)\right) \mathrm{d} h \mathrm{~d} x \mathrm{~d} t \\
=-\int_{0}^{t^{*}} \int_{\Omega}^{b} p\left(v^{*}\right) A\left(y(v)-y\left(v^{*}\right)\right) \mathrm{d} x \mathrm{~d} t \\
-\int_{a} \int_{\Omega}^{t^{*}} \int_{0} p\left(x, t ; v^{*}\right) c(x, t) \\
=(x)
\end{aligned}
$$




$$
\begin{aligned}
& \times\left(y(x, t-h ; v)-y\left(x, t-h ; v^{*}\right)\right) \mathrm{d} t \mathrm{~d} x \mathrm{~d} h \\
& =-\int_{0}^{t^{*}} \int_{\Omega} p\left(v^{*}\right) A\left(y(v)-y\left(v^{*}\right)\right) \mathrm{d} x \mathrm{~d} t \\
& -\int_{a}^{b} \int_{\Omega}^{t^{*}-h} \int_{-h}^{t^{\prime}} p\left(x, t^{\prime}+h ; v^{*}\right) c\left(x, t^{\prime}+h\right) \\
& \times\left(y\left(x, t^{\prime} ; v\right)-y\left(x, t^{\prime} ; v^{*}\right)\right) \mathrm{d} t^{\prime} \mathrm{d} x \mathrm{~d} h \\
& =-\int_{0}^{t^{*}} \int_{\Omega} p\left(v^{*}\right) A\left(y(v)-y\left(v^{*}\right)\right) \mathrm{d} x \mathrm{~d} t \\
& -\int_{a}^{b} \int_{\Omega} \int_{-h}^{0} p\left(x, t^{\prime}+h ; v^{*}\right) c\left(x, t^{\prime}+h\right) \\
& \times\left(y\left(x, t^{\prime} ; v\right)-y\left(x, t^{\prime} ; v^{*}\right)\right) \mathrm{d} t^{\prime} \mathrm{d} x \mathrm{~d} h \\
& -\int_{a}^{b} \int_{\Omega}^{t^{*}} \int_{0}^{-b} p\left(x, t^{\prime}+h ; v^{*}\right) c\left(x, t^{\prime}+h\right) \\
& \times\left(y\left(x, t^{\prime} ; v\right)-y\left(x, t^{\prime} ; v^{*}\right)\right) \mathrm{d} t^{\prime} \mathrm{d} x \mathrm{~d} h \\
& -\int_{a}^{b} \int_{\Omega} \int_{t^{*}-b}^{t^{*}-h} p\left(x, t^{\prime}+h ; v^{*}\right) c\left(x, t^{\prime}+h\right) \\
& \times\left(y\left(x, t^{\prime} ; v\right)-y\left(x, t^{\prime} ; v^{*}\right)\right) \mathrm{d} t^{\prime} \mathrm{d} x \mathrm{~d} h \\
& =-\int_{0}^{t^{*}} \int_{\Omega} p\left(v^{*}\right) A\left(y(v)-y\left(v^{*}\right)\right) \mathrm{d} x \mathrm{~d} t \\
& -\int_{a}^{b} \int_{\Omega} \int_{-h}^{0} p\left(x, t^{\prime}+h ; v^{*}\right) c\left(x, t^{\prime}+h\right) \\
& \times\left(y\left(x, t^{\prime} ; v\right)-y\left(x, t^{\prime} ; v^{*}\right)\right) \mathrm{d} t^{\prime} \mathrm{d} x \mathrm{~d} h \\
& -\int_{a}^{b} \int_{\Omega} \int_{0}^{t^{*}-b} p\left(x, t^{\prime}+h ; v^{*}\right) c\left(x, t^{\prime}+h\right) \\
& \times\left(y\left(x, t^{\prime} ; v\right)-y\left(x, t^{\prime} ; v^{*}\right)\right) \mathrm{d} t^{\prime} \mathrm{d} x \mathrm{~d} h \\
& -\int_{a}^{t^{*}-t} \int_{\Omega} \int_{t^{*}-b}^{t^{*}-a} p\left(x, t^{\prime}+h ; v^{*}\right) c\left(x, t^{\prime}+h\right) \\
& \times\left(y\left(x, t^{\prime} ; v\right)-y\left(x, t^{\prime} ; v^{*}\right)\right) \mathrm{d} t^{\prime} \mathrm{d} x \mathrm{~d} h .
\end{aligned}
$$

The second integral on the right-hand side of (44), in view of Green's formula, can be expressed as

$$
\begin{aligned}
& \int_{0}^{t^{*}} \int_{\Omega} A^{*} p\left(v^{*}\right)\left(y(v)-y\left(v^{*}\right)\right) \mathrm{d} x \mathrm{~d} t \\
& =\int_{0}^{t^{*}} \int_{\Omega} p\left(v^{*}\right) A\left(y(v)-y\left(v^{*}\right)\right) \mathrm{d} x \mathrm{~d} t
\end{aligned}
$$

$$
\begin{aligned}
& +\int_{0}^{t^{*}} \int_{\Gamma} p\left(v^{*}\right)\left(\frac{\partial y(v)}{\partial \eta_{A}}-\frac{\partial y\left(v^{*}\right)}{\partial \eta_{A}}\right) \mathrm{d} \Gamma \mathrm{d} t \\
& -\int_{0}^{t^{*}} \int_{\Gamma} \frac{\partial p\left(v^{*}\right)}{\partial \eta_{A^{*}}}\left(y(v)-y\left(v^{*}\right)\right) \mathrm{d} \Gamma \mathrm{d} t .
\end{aligned}
$$

Using the boundary condition (9), the second component on the right-hand side of (46) can be written as

$$
\begin{aligned}
& \int_{0}^{t^{*}} \int_{\Gamma} p\left(v^{*}\right)\left[\frac{\partial y(v)}{\partial \eta_{A}}-\frac{\partial y\left(v^{*}\right)}{\partial \eta_{A}}\right] \mathrm{d} \Gamma \mathrm{d} t \\
& =\int_{0}^{t^{*}} \int_{\Gamma} p\left(x, t ; v^{*}\right)\left(\int_{a}^{b} d(x, t)\right. \\
& \left.\times\left(y(x, t-h ; v)-y\left(x, t-h ; v^{*}\right)\right) \mathrm{d} h\right) \mathrm{d} \Gamma \mathrm{d} t \\
& +\int_{0}^{t^{*}} \int_{\Gamma} p\left(x, t ; v^{*}\right)\left(v-v^{*}\right) \mathrm{d} \Gamma \mathrm{d} t \\
& =\int_{0}^{t^{*}} \int_{\Gamma} \int_{a}^{b} p\left(x, t ; v^{*}\right) d(x, t) \\
& \times\left(y(x, t-h ; v)-y\left(x, t-h ; v^{*}\right)\right) \mathrm{d} h \mathrm{~d} \Gamma \mathrm{d} t \\
& +\int_{0}^{t^{*}} \int_{\Gamma} p\left(x, t ; v^{*}\right)\left(v-v^{*}\right) \mathrm{d} \Gamma \mathrm{d} t \\
& =\int_{a}^{b} \int_{\Gamma} \int_{0}^{t^{*}} p\left(x, t ; v^{*}\right) d(x, t) \\
& \times\left(y(x, t-h ; v)-y\left(x, t-h ; v^{*}\right)\right) \mathrm{d} t \mathrm{~d} \Gamma \mathrm{d} h \\
& +\int_{0}^{t^{*}} \int_{\Gamma} p\left(x, t ; v^{*}\right)\left(v-v^{*}\right) \mathrm{d} \Gamma \mathrm{d} t \\
& =\int_{a}^{b} \int_{\Gamma}^{t^{*}} \int_{-h}^{-h} p\left(x, t^{\prime}+h ; v^{*}\right) d\left(x, t^{\prime}+h\right) \\
& \times\left(y\left(x, t^{\prime} ; v\right)-y\left(x, t^{\prime} ; v^{*}\right)\right) \mathrm{d} t^{\prime} \mathrm{d} \Gamma \mathrm{d} h \\
& +\int_{0}^{t^{*}} \int_{\Gamma} p\left(x, t ; v^{*}\right)\left(v-v^{*}\right) \mathrm{d} \Gamma \mathrm{d} t \\
& =\int_{a}^{b} \int_{\Gamma} \int_{-h}^{0} p\left(x, t^{\prime}+h ; v^{*}\right) d\left(x, t^{\prime}+h\right) \\
& \times\left(y\left(x, t^{\prime} ; v\right)-y\left(x, t^{\prime} ; v^{*}\right)\right) \mathrm{d} t^{\prime} \mathrm{d} \Gamma \mathrm{d} h
\end{aligned}
$$




$$
\begin{aligned}
& +\int_{a}^{b} \int_{\Gamma}^{t^{*}-b} \int_{0}^{t^{\prime}} p\left(x, t^{\prime}+h ; v^{*}\right) d\left(x, t^{\prime}+h\right) \\
& \times\left(y\left(x, t^{\prime} ; v\right)-y\left(x, t^{\prime} ; v^{*}\right)\right) \mathrm{d} t^{\prime} \mathrm{d} \Gamma \mathrm{d} h \\
& +\int_{a}^{b} \int_{\Gamma} \int_{t^{*}-b}^{t^{*}-h} p\left(x, t^{\prime}+h ; v^{*}\right) d\left(x, t^{\prime}+h\right) \\
& \times\left(y\left(x, t^{\prime} ; v\right)-y\left(x, t^{\prime} ; v^{*}\right)\right) \mathrm{d} t^{\prime} \mathrm{d} \Gamma \mathrm{d} h \\
& +\int_{0}^{t^{*}} \int_{\Gamma} p\left(x, t ; v^{*}\right)\left(v-v^{*}\right) \mathrm{d} \Gamma \mathrm{d} t \\
& =\int_{a}^{b} \int_{\Gamma} \int_{-h}^{0} p\left(x, t^{\prime}+h ; v^{*}\right) d\left(x, t^{\prime}+h\right) \\
& \times\left(y\left(x, t^{\prime} ; v\right)-y\left(x, t^{\prime} ; v^{*}\right)\right) \mathrm{d} t^{\prime} \mathrm{d} \Gamma \mathrm{d} h \\
& +\int_{a}^{b} \int_{\Gamma}^{t^{*}-b} \int_{0} p\left(x, t^{\prime}+h ; v^{*}\right) d\left(x, t^{\prime}+h\right) \\
& \times\left(y\left(x, t^{\prime} ; v\right)-y\left(x, t^{\prime} ; v^{*}\right)\right) \mathrm{d} t^{\prime} \mathrm{d} \Gamma \mathrm{d} h \\
& +\int_{a}^{t^{*}-t} \int_{\Gamma} \int_{t^{*}-b}^{t^{*}-a} p\left(x, t^{\prime}+h ; v^{*}\right) d\left(x, t^{\prime}+h\right) \\
& \times\left(y\left(x, t^{\prime} ; v\right)-y\left(x, t^{\prime} ; v^{*}\right)\right) \mathrm{d} t^{\prime} \mathrm{d} \Gamma \mathrm{d} h \\
& +\int_{0}^{t^{*}} \int_{\Gamma} p\left(x, t ; v^{*}\right)\left(v-v^{*}\right) \mathrm{d} \Gamma \mathrm{d} t .
\end{aligned}
$$

The last component in (46) can be rewritten as

$$
\begin{aligned}
& \int_{0}^{t^{*}} \int_{\Gamma} \frac{\partial p\left(v^{*}\right)}{\partial \eta_{A^{*}}}\left(y(v)-y\left(v^{*}\right)\right) \mathrm{d} \Gamma \mathrm{d} t \\
& =\int_{0}^{t^{*}-b} \int_{\Gamma} \frac{\partial p\left(v^{*}\right)}{\partial \eta_{A^{*}}}\left(y(v)-y\left(v^{*}\right)\right) \mathrm{d} \Gamma \mathrm{d} t \\
& +\int_{t^{*}-b}^{t^{*}-a} \frac{\partial p\left(v^{*}\right)}{\partial \eta_{A^{*}}}\left(y(v)-y\left(v^{*}\right)\right) \mathrm{d} \Gamma \mathrm{d} t \\
& +\int_{t^{*}-a} \int_{\Gamma} \frac{\partial p\left(v^{*}\right)}{\partial \eta_{A^{*}}}\left(y(v)-y\left(v^{*}\right)\right) \mathrm{d} \Gamma \mathrm{d} t .
\end{aligned}
$$

Substituting (47) and (48) into (46) and then (45) and (46) into (44), we obtain

$$
\begin{aligned}
& \int_{\Omega}\left(z_{d}-y\left(t^{*} ; v^{*}\right)\right)\left(y\left(t^{*} ; v\right)-y\left(t^{*} ; v^{*}\right)\right) \mathrm{d} x \\
& =-\int_{0}^{t^{*}} \int_{\Omega} p\left(v^{*}\right) A\left(y(v)-y\left(v^{*}\right)\right) \mathrm{d} x \mathrm{~d} t
\end{aligned}
$$

$-\int_{a}^{b} \int_{\Omega} \int_{-h}^{0} c(x, t+h) p\left(x, t+h ; v^{*}\right)$

$\times\left(y(x, t ; v)-y\left(x, t ; v^{*}\right)\right) \mathrm{d} t \mathrm{~d} x \mathrm{~d} h$

$-\int_{a}^{b} \int_{\Omega}^{t^{*}-b} \int_{0}^{b} c(x, t+h) p\left(x, t+h ; v^{*}\right)$

$\times\left(y(x, t ; v)-y\left(x, t ; v^{*}\right)\right) \mathrm{d} t \mathrm{~d} x \mathrm{~d} h$

$-\int_{a}^{t^{*}-t} \int_{\Omega} \int_{t^{*}-b}^{t^{*}-a} c(x, t+h) p\left(x, t+h ; v^{*}\right)$

$\times\left(y(x, t ; v)-y\left(x, t ; v^{*}\right)\right) \mathrm{d} t \mathrm{~d} x \mathrm{~d} h$

$+\int_{0}^{t^{*}} \int_{\Omega} p\left(v^{*}\right) A\left(y(v)-y\left(v^{*}\right)\right) \mathrm{d} x \mathrm{~d} t$

$+\int_{a}^{b} \int_{\Gamma} \int_{-h}^{0} d(x, t+h) p\left(x, t+h ; v^{*}\right)$

$\times\left(y(x, t ; v)-y\left(x, t ; v^{*}\right)\right) \mathrm{d} t \mathrm{~d} \Gamma \mathrm{d} h$

$+\int_{a}^{b} \int_{\Gamma}^{t^{*}-b} \int_{0}^{b} d(x, t+h) p\left(x, t+h ; v^{*}\right)$

$\times\left(y(x, t ; v)-y\left(x, t ; v^{*}\right)\right) \mathrm{d} t \mathrm{~d} \Gamma \mathrm{d} h$

$+\int_{a}^{t^{*}-t} \int_{\Gamma} \int_{t^{*}-b}^{t^{*}-a} d(x, t+h) p\left(x, t+h ; v^{*}\right)$

$\times\left(y(x, t ; v)-y\left(x, t ; v^{*}\right)\right) \mathrm{d} t \mathrm{~d} \Gamma \mathrm{d} h$

$+\int_{0}^{t^{*}} \int_{\Gamma} p\left(x, t ; v^{*}\right)\left(v-v^{*}\right) \mathrm{d} \Gamma \mathrm{d} t$

$-\int_{0}^{t^{*}-b} \int_{\Gamma} \frac{\partial p\left(v^{*}\right)}{\partial \eta_{A^{*}}}\left(y(x, t ; v)-y\left(x, t ; v^{*}\right)\right) \mathrm{d} \Gamma \mathrm{d} t$

$-\int_{t^{*}-b}^{t^{*}-a} \int_{\Gamma} \frac{\partial p\left(v^{*}\right)}{\partial \eta_{A^{*}}}\left(y(x, t ; v)-y\left(x, t ; v^{*}\right)\right) \mathrm{d} \Gamma \mathrm{d} t$

$-\int_{t^{*}-a}^{t^{*}} \int_{\Gamma} \frac{\partial p\left(v^{*}\right)}{\partial \eta_{A^{*}}}\left(y(x, t ; v)-y\left(x, t ; v^{*}\right)\right) \mathrm{d} \Gamma \mathrm{d} t$

$+\int_{a}^{b} \int_{\Omega}^{t^{*}-b} \int_{0}^{b} c(x, t+h) p\left(x, t+h ; v^{*}\right)$

$\times\left(y(x, t ; v)-y\left(x, t ; v^{*}\right)\right) \mathrm{d} t \mathrm{~d} x \mathrm{~d} h$

$+\int_{a}^{t^{*}-t} \int_{\Omega} \int_{t^{*}-b}^{t^{*}-a} c(x, t+h) p\left(x, t+h ; v^{*}\right)$

$\times\left(y(x, t ; v)-y\left(x, t ; v^{*}\right)\right) \mathrm{d} t \mathrm{~d} x \mathrm{~d} h$. 
Then, using the fact that $y(x, t ; v)=y\left(x, t ; v^{*}\right)=$ $\Phi_{0}(x, t)$ for $x \in \Omega$ and $t \in[-b, 0)$ and $y(x, t ; v)=$ $y\left(x, t ; v^{*}\right)=\Psi_{0}(x, t)$ for $x \in \Gamma$ and $t \in[-b, 0)$, we obtain

$$
\begin{gathered}
\int_{\Omega}\left(z_{d}-y\left(t^{*} ; v^{*}\right)\right)\left(y\left(t^{*} ; v\right)-y\left(t^{*} ; v^{*}\right)\right) \mathrm{d} x \\
=\int_{0}^{t^{*}} \int_{\Gamma} p\left(v^{*}\right)\left(v-v^{*}\right) \mathrm{d} \Gamma \mathrm{d} t .
\end{gathered}
$$

Substituting (50) into (24) gives

$$
\int_{0}^{t^{*}} \int_{\Gamma} p\left(v^{*}\right)\left(v-v^{*}\right) \mathrm{d} \Gamma \mathrm{d} t \leq 0, \quad \forall v \in U .
$$

The foregoing result is now summarized.

Theorem 4. The optimal control $v^{*}$ is characterized by the condition (51). Moreover, in a particular case we have

$$
v^{*}(x, t)=\operatorname{sign}\left(p\left(x, t ; v^{*}\right)\right), \quad x \in \Gamma, \quad t \in\left(0, t^{*}\right)
$$

whenever $p\left(v^{*}\right)$ is nonzero.

This property leads to the following result:

Theorem 5. If the functions $c(x, t)$ and $d(x, t)$ are analytic and $\Omega$ has an analytic boundary $\Gamma$, then there exists a unique optimal control for the mixed initial-boundary value problem (6)-(10). Moreover, the optimal control is bang-bang, i.e., $\left|v^{*}(x, t)\right| \equiv 1$, almost everywhere and the unique solution of (6)-(10), (35)-(41), (52).

The idea of the proof of Theorem 5 is the same as in the case of Theorem 3.4 in (Kowalewski and Krakowiak, 2000).

\section{Conclusions and perspectives}

The results presented in the paper can be treated as a generalization of the results obtained by (Knowles, 1978; Kowalewski and Krakowiak, 1994; Kowalewski and Krakowiak, 2000; Kowalewski and Krakowiak, 2006) onto the case of the time-optimal boundary control of infinite order parabolic systems with deviating arguments appearing in the integral form both in state equations and in boundary conditions. Such parabolic systems constitute a generalization of the mathematical model for a plasma control process.

We considered a different type of control, namely, the control function defined at the boundary of a system spatial domain. Sufficient conditions for the existence of a unique solution of such parabolic equations with Neumann boundary conditions are proved (Lemma1 1 and Theorem 11. The optimal control is characterized by using the adjoint equation (Theorems 2 and 3). The uniqueness and bang-bang properties of the optimal control are proved (Theorems 4 and 5).

The condition (23) plays a fundamental role in controllability problems for time-delay infinite order parabolic systems. With regard to the controllability assumption (23) we can investigate the exact controllability problem for the infinite order parabolic system (6)-(10).

In this paper we considered the time-optimal boundary control problem for infinite order parabolic systems with nonhomogeneous Neumann boundary conditions. We can also consider the analogous minimum time problem for such systems with non-homogeneous Dirichlet boundary conditions.

Finally, we can consider a time-optimal control problem for discrete time delay distributed parameter systems. The ideas mentioned above will be developed in forthcoming papers.

\section{Acknowledgements}

The first author would like to thank Professor A.J. Pritchard, the Director of the Control Theory Centre, for the invitation and the hospitality at the Department of Mathematics, the University of Warwick. He would like to express his gratitude to Professor Pritchard for many discussions and many valuable suggestions during the author's stay at the Control Theory Centre, the University of Warwick.

The research presented here was carried out within the research programme of the AGH University of Science and Technology, no. 10.10.120.31.

\section{References}

Choquet, G. (1969). Lectures on Analysis, Vol.2, W.A. Benjamin, New York.

Dubinskii, J. A. (1975). Sobolev spaces of infinite order and behavior of solution of some boundary value problems with unbounded increase of the order of the equation, Matiematiczeskii Sbornik 98: 163-184, (in Russian).

Dubinskii, J. A. (1976). Non-trivality of Sobolev spaces of infinite order for a full Euclidean space and a torus, Matiematiczeskii Sbornik 100: 436-446, (in Russian).

Dubinskii, J. A. (1981). About one method for solving partial differential equations, Doklady Akademii Nauk SSSR 258: 780-784, (in Russian).

Dunford, N. and Schwartz, J. (1958). Linear Operators, Vol. 1, John Wiley and Sons, New York.

El-Saify, H. A. (2005). Optimal control of $n \times n$ parabolic lag system involving time lag, IMA Journal of Mathematical Control and Information 22(3): 240-250.

El-Saify, H. A. (2006). Optimal boundary control problem for $n \times n$ infinite order parabolic lag system, IMA Journal of Mathematical Control and Information 23(4): 433-445. 
Knowles, G. (1978). Time optimal control of parabolic systems with boundary conditions involving time delays, Journal of Optimization Theory and Applications 25(4): 563-574.

Kowalewski, A. (1988). Boundary control of distributed parabolic system with boundary condition involving a timevarying lag, International Journal of Control 48(6): 22332248.

Kowalewski, A. (1990a). Feedback control for a distributed parabolic system with boundary condition involving a timevarying lag, IMA Journal of Mathematical Control and Information 7(2): 143-157.

Kowalewski, A. (1990b). Optimal control of distributed parabolic systems involving time lags, IMA Journal of Mathematical Control and Information 7(4): 375-393.

Kowalewski, A. (1993). Optimal control of parabolic systems with time-varying lags, IMA Journal of Mathematical Control and Information 10(2): 113-129.

Kowalewski, A. (1998). Optimal control of distributed parabolic systems with multiple time-varying lags, International Journal of Control 69(3): 361-381.

Kowalewski, A. (1999). Optimization of parabolic systems with deviating arguments, International Journal of Control 72(11): 947-959.

Kowalewski, A. (2001). Optimal Control of Infinite Dimensional Distributed Parameter Systems with Delays, University of Mining and Metallurgy Press, Cracow.

Kowalewski, A. and Duda, J. (1992). On some optimal control problem for a parabolic system with boundary condition involving a time-varying lag, IMA Journal of Mathematical Control and Information 9(2): 131-146.
Kowalewski, A. and Krakowiak, A. (1994). Time-optimal control of parabolic time lag system, Applied Mathematics and Computer Science 4(1): 19-28.

Kowalewski, A. and Krakowiak, A. (2000). Time-optimal control of parabolic system with time lags given in the integral form, IMA Journal of Mathematical Control and Information 17(3): 209-225.

Kowalewski, A. and Krakowiak, A. (2006). Time-optimal boundary control of a parabolic system with time lags given in the integral form, International Journal of Applied Mathematics and Computer Science 16(3): 287-295.

Lions, J. (1971). Optimal Control of Systems Governed by Partial Differential Equations, Springer-Verlag, BerlinHeidelberg.

Lions, J. and Magenes, E. (1972). Non-Homogeneous Boundary Value Problems and Applications, Vols. 1 and 2, SpringerVerlag, Berlin-Heidelberg.

Wang, P. K. C. (1975). Optimal control of parabolic systems with boundary conditions involving time delays, SIAM Journal on Control 13(2): 274-293.

Received: 7 May 2007

Revised: 30 September 2007

Re-revised: 18 March 2008 